\title{
Adaptive testing with the GGUM-RANK multidimensional forced choice model: Comparison of pair, triplet, and tetrad scoring
}

\author{
Seang-Hwane Joo ${ }^{1} \cdot$ Philseok Lee $^{2} \cdot$ Stephen Stark ${ }^{1}$
}

Published online: 24 July 2019

(C) The Psychonomic Society, Inc. 2019

\begin{abstract}
Likert-type measures have been criticized in psychological assessment because they are vulnerable to response biases, including central tendency, acquiescence, leniency, halo, and socially desirable responding. As an alternative, multidimensional forced choice (MFC) testing has been proposed to address these concerns. A number of researchers have developed item response theory (IRT) models for MFC data and have examined latent trait estimation with tests of different dimensionality and length. Research has also explored the advantages of computerized adaptive testing (CAT) with MFC pair tests having as many as 25 dimensions, but there have been no published studies on CAT with MFC triplets or tetrads. Thus, in this research we aimed to address that issue. We used recently developed item information functions for an MFC ranking model to compare the benefits of CAT with MFC pair, triplet, and tetrad tests. A simulation study showed that CAT substantially outperformed nonadaptive testing for latent trait estimation across MFC formats. More importantly, CAT with MFC pairs provided estimation accuracy similar to or better than that from tests of equivalent numbers of nonadaptive MFC triplets. On the basis of these findings, implications and recommendations are further discussed for constructing MFC measures to use in psychological contexts.
\end{abstract}

Keywords Multidimensional forced choice · Computerized adaptive testing · GGUM-RANK · Ideal point

In the field of psychological testing, forced choice measures have been suggested to reduce the response biases associated with Likert-type measures, including central tendency, acquiescence, leniency, halo, and socially desirable responding. Toward that end, a variety of item response theory (IRT) models for forced choice responses have been developed (e.g., Andrich, 1995; Brown \& Maydeu-Olivares, 2011; Morillo et al., 2016; Stark, Chernyshenko, \& Drasgow, 2005; Wang, Qiu, Chen, Ro, \& Jin, 2017; Zinnes \& Griggs, 1974). There has also been some research on forced choice computerized adaptive testing (CAT), but it has focused exclusively on pairwise preference models. For example, Borman et al. (2001) examined reliability, perceived favorability, and effects of halo and leniency errors in an employee performance appraisal study comparing a unidimensional forced-choice CAT (Stark, Chernyshenko, \& Guenole, 2011; Stark \& Drasgow, 1998; Zinnes \& Griggs, 1974) with other types of rating scales.

Seang-Hwane Joo

sjoo@mail.usf.edu

1 University of South Florida, Tampa, FL, USA

2 George Mason University, Fairfax, VA, USA
Subsequent research examined the viability of that methodology for personality assessment in the U.S. Navy (Houston, Borman, Farmer, \& Bearden, 2006; Underhill, Lords, \& Bearden, 2006) as well as in organizational settings with emphasis on test administration time (Kantrowitz \& Tuzinski, 2010) and test security (Kantrowitz, Dawson, \& Fetzer, 2011).

For further resistance to faking in high-stakes testing environments, Stark et al. (2005) also developed the multiunidimensional pairwise preference (MUPP) model, which matches statements reflecting different psychological dimensions on extremity and social desirability, and they conducted a series of simulations to explore the effects of test dimensionality, length, latent trait correlations, and other test design specifications on the accuracy and precision of latent trait estimates with nonadaptive and adaptive item selection (Stark, Chernyshenko, Drasgow, \& White, 2012). Since then, the number of applications using forced choice pairwise preference CATs has grown considerably (e.g., Computer Adaptive Rating Scales [CARS; Borman et al., 2001]; Navy Computer Adaptive Personality Scales [NCAPS; Houston et al., 2006]; Global Personality Inventory-Adaptive [GPI-A; CEB, 2010]; Tailored Adaptive Personality Assessment System [TAPAS; Drasgow et al., 2012; Stark et al., 2014]; Adaptive Employee Personality Test [Adept-15; Aon Hewitt, 2015]). 
However, research has yet to explore the benefits of CAT with more complex formats, such as forced choice triplets (three-alternative) and tetrads (four-alternative). We believe this gap in the literature exists for two reasons. Until recently MFC triplet and tetrad measures were scored with heuristics rooted in classical test theory (White \& Young, 1998) or unidimensional IRT (McCloy, Heggestad, \& Reeve, 2005; Stark, Chernyshenko, Lee, et al., 2011), rather than formal models for comparative judgments involving 2 to $K$ response alternatives. The Thurstonian IRT model (Brown \& MaydeuOlivares, 2011) and the more recent PICK (choose one of $K$ alternatives), RANK (rank $K$ alternatives), and MOLE (choose "most preferred" and "least preferred" from $K$ alternatives) generalizations of the MUPP (de la Torre, Ponsoda, Leenen, \& Hontangas, 2012; Hontangas et al., 2015) were breakthroughs that provided a pathway forward. Yet, applications and extensions with more than two response alternatives (e.g., Anguiano-Carrasco, MacCann, Geiger, Seybert, \& Roberts, 2015; Guenole, Brown, \& Cooper, 2018; P. Lee, Joo, \& Lee, 2019; P. Lee, Joo, Stark, \& Chernyshenko, 2019; Pavlov, Maydeu-Olivares, \& Fairchild, 2019; Seybert, 2013) have been limited to nonadaptive testing, by either estimation software that is not conducive to CAT or, for the RANK model specifically, the unavailability of item information equations needed for adaptive item selection.

To address these issues and provide a foundation for CAT research with the RANK model, now referred to as GGUMRANK (because it uses the Generalized Graded Unfolding Model [GGUM; Roberts, Donoghue, \& Laughlin, 2000] to compute alternative endorsement probabilities), Joo, Lee, and Stark (2018) derived the necessary item information equations and showed that GGUM-RANK triplet and tetrad measures provide substantially higher test information and marginal reliability than similarly discriminating pairwise preference measures of equal length. This finding, which accords with parameter estimation results for triplets in Lee et al. (2019), has important implications for field applications. Although MFC pairwise preference CATs have been shown to provide markedly better estimation than nonadaptive tests of equal length (Stark et al., 2012; Stark et al., 2014), the overall number of items administered must still be fairly high when many dimensions are assessed (e.g., 100 items to measure ten dimensions). Moreover, because answering many items in related content areas could lead to momentary lapses in examinee attention or effort, it might be better to use a smaller number of triplets or tetrads to accomplish the same objective. It is, therefore, important to know how latent trait estimation compares for MFC pair, triplet, and tetrad CATs, as well as how such CATs perform relative to similarly configured nonadaptive tests. That brings us to the purpose of this study.

In the sections that follow, we present a Monte Carlo study that compared latent trait estimation with MFC pair, triplet, and tetrad tests using nonadaptive and adaptive item selection based on the GGUM-RANK model. For this initial foray into MFC rank CAT, we focused on low dimensionality assessments. However, the methods and findings should provide a foundation for future research with other formats (e.g., MOLE and PICK from $K$ alternatives) and tests of higher dimensionality.

\section{The GGUM-RANK model}

The GGUM-RANK model (Hontangas et al., 2015; P. Lee, Joo, Stark, \& Chernyshenko, 2019) is an extension of the MUPP (Stark et al., 2005), which assumes that a respondent independently evaluates the statements composing a pairwise preference item until a preference is reached. A preference entails agreeing with one statement and disagreeing with the other. MUPP response probabilities are computed as follows:

$$
\begin{aligned}
& P_{(s>t)}\left(\theta_{d_{s}}, \theta_{d_{t}}\right) \\
& =\frac{P_{s t}(1,0)}{P_{s t}(1,0)+P_{s t}(0,1)} \approx \frac{P_{s}(1) P_{t}(0)}{P_{s}(1) P_{t}(0)+P_{s}(0) P_{t}(1)},
\end{aligned}
$$

where $P_{(s>t)}\left(\theta_{d_{s}}, \theta_{d_{t}}\right)$ represents the probability of preferring statement $s$ to statement $t$, given a respondent's scores $\left(\theta_{d_{s}}, \theta_{d_{t}}\right.$ ) on the dimensions represented by the statements; $P_{s t}(1,0)$ represents the joint probability of agreeing with statement $s$ and disagreeing with statement $t$; and $P_{s t}(0,1)$ represents the joint probability of disagreeing with statement $s$ and agreeing with statement $t$. By assuming the statements are evaluated independently, the joint probabilities can be factored and the $P(1)$ and $P(0)$ terms on the right computed using a suitable unidimensional model. To compute the probability of agreeing with a statement, Stark et al. chose the dichotomous GGUM (Roberts et al., 2000) in accordance with research that examined the fit of several dominance and ideal point models to personality data (Chernyshenko, Stark, Chan, Drasgow, \& Williams, 2001; Stark, Chernyshenko, Drasgow, \& Williams, 2006). The probability of agreeing with a statement, $P(1)$, depends on a respondent's trait level $(\theta)$ and the statement's discrimination $(\alpha)$, location $(\delta)$, and threshold $(\tau)$ parameters, respectively:

$$
\begin{aligned}
P(1) & =P(Z=1 \mid \theta) \\
& =\frac{\exp (\alpha[(\theta-\delta)-\tau])+\exp (\alpha[2(\theta-\delta)-\tau])}{\gamma},
\end{aligned}
$$

where $\gamma=1+\exp (\alpha[3(\theta-\delta)])+\exp (\alpha[2(\theta-\delta)-\tau])+$ $\exp (\alpha[(\theta-\delta)-\tau])$. The probability of disagreeing with the statement is $P(0)=1-P(1)$.

The MUPP model was later generalized to handle the more complex MFC formats and instruction sets explored in 
practice (de la Torre et al., 2012; Hontangas et al., 2015). Three extensions of the MUPP model were proposed, specifically for the MFC tetrad format, and they are referred to as PICK, MOLE, and RANK, respectively. The PICK model applies when respondents are asked to choose the "most preferred" statement in an MFC item. The MOLE model applies when respondents must choose the "most preferred" (MO) and "least preferred" (LE) statements. And the RANK model applies when respondents must rank the statements from most preferred to least preferred. Hontangas et al. compared latent trait estimation accuracy for the PICK, MOLE, and RANK models with MFC tetrads and found that the RANK formulation provided the best results. Therefore, we focused on the RANK version of their model (i.e., GGUM-RANK) for our study of adaptive versus nonadaptive testing with pairs, triplets, and tetrads.

To illustrate the GGUM-RANK probability equations, consider an MFC tetrad item as an example. An MFC tetrad consists of four statements, so there are $4 !=24$ possible rank order responses. In accordance with Luce (1959/2005), the probability of a particular ranking is obtained by multiplying a series of conditional (PICK) probabilities (de la Torre et al., 2012). For example, the probability of the ranking $A>B>C$ $>$ D (i.e., statement $\mathrm{A}$ is most preferred, then $\mathrm{B}$ and then $\mathrm{C}$, and $\mathrm{D}$ is the least preferred) is the product of the probabilities of picking statement $A$ from the set of four statements, A, B, C, and $\mathrm{D}$; picking statement $\mathrm{B}$ from the remaining set of three statements, B, C, and D; and picking statement $\mathrm{C}$ from the remaining pair, $\mathrm{C}$ and $\mathrm{D}$. Letting the respondents' trait scores on the dimensions represented by the statements A, B, C, and $\mathrm{D}$ be $\theta_{d_{A}}, \theta_{d_{B}}, \theta_{d_{C}}$, and $\theta_{d_{D}}$, respectively, the GGUM-RANK probability of the ranking $\mathrm{A}>\mathrm{B}>\mathrm{C}>\mathrm{D}$ is

$$
\begin{aligned}
& P_{(A>B>C>D)}\left(\theta_{d_{A}}, \theta_{d_{B}}, \theta_{d_{C}}, \theta_{d_{D}}\right) \\
& \quad=P_{(A \mid A, B, C, D)} \times P_{(B \mid B, C, D)} \times P_{(C \mid C, D)},
\end{aligned}
$$

where the PICK probability $P_{(A \mid A, B, C, D)}$ in Eq. 3 is defined as

$P_{(A \mid A, B, C, D)}=\frac{P_{A, B, C, D}(1,0,0,0)}{P_{A, B, C, D}(1,0,0,0)+P_{A, B, C, D}(0,1,0,0)+P_{A, B, C, D}(0,0,1,0)+P_{A, B, C, D}(0,0,0,1)}$,

and

$P_{A, B, C, D}(1,0,0,0)=P_{A}(1) P_{B}(0) P_{C}(0) P_{D}(0)$,

$P_{A, B, C, D}(0,1,0,0)=P_{A}(0) P_{B}(1) P_{C}(0) P_{D}(0)$,

$P_{A, B, C, D}(0,0,1,0)=P_{A}(0) P_{B}(0) P_{C}(1) P_{D}(0)$,
$P_{A, B, C, D}(0,0,0,1)=P_{A}(0) P_{B}(0) P_{C}(0) P_{D}(1)$.

Note that $P_{X}(1)$ is the GGUM probability of agreeing with statement $X$, shown in Eq. 2, and $P_{X}(0)=1-P_{X}(1)$. Similarly, the PICK probabilities $P_{(B \mid B, C, D)}$ and $P_{(C \mid C, D)}$ in Eq. 3 are:

$P_{(B \mid B, C, D)}=\frac{P_{B}(1) P_{C}(0) P_{D}(0)}{P_{B}(1) P_{C}(0) P_{D}(0)+P_{B}(0) P_{C}(1) P_{D}(0)+P_{B}(0) P_{C}(0) P_{D}(1)}$,

$P_{(C \mid C, D)}=\frac{P_{C}(1) P_{D}(0)}{P_{C}(1) P_{D}(0)+P_{C}(0) P_{D}(1)}$.

The same logic applies to MFC triplets and pairs. With an MFC triplet, there are $3 !=6$ possible ways a respondent can rank the three statements, and with an MFC pair, there are $2 !=$ 2 ways a respondent can rank the two statements. Note that Eq. 10, the probability of the ranking $\mathrm{C}>\mathrm{D}$, is equivalent to the MUPP probability (Eq. 1).

\section{Information functions}

Joo et al. (2018) derived GGUM-RANK item information functions following definitions and procedures for polytomous (Baker \& Kim, 2004) and multidimensional IRT models (Segall, 1996). Their general equations are shown below, and readers are referred to Joo et al. (2018) for more detailed information about the GGUM-RANK information function derivation and overall item information (OII) and overall test information (OTI) indices.

Let $I_{j}(\boldsymbol{\theta})$ represents the item information function (IIF) for MFC item $j$ :

$I_{j}(\theta)=\sum_{k=1}^{m_{j}} I_{j k}(\theta) P_{j k}(\theta)$,

where $P_{j k}(\theta)$ is the probability of choosing or endorsing ranking $k\left(k=1,2, \ldots, m_{j}\right)$ at trait level $\boldsymbol{\theta}$, and $I_{j k}(\boldsymbol{\theta})$ is Fisher information associated with ranking $k$ at trait level $\boldsymbol{\theta}$ :

$I_{j k}(\boldsymbol{\theta})=-E\left[\frac{\partial^{2} \ln P_{j k}(\boldsymbol{\theta})}{\partial \boldsymbol{\theta} \partial \boldsymbol{\theta}^{T}}\right]$. 
The $(u, v)$ th element of the Fisher information matrix is:

$\left[I_{j k}(\boldsymbol{\theta})\right]_{u, v}=-E\left[\frac{\partial^{2} \ln P_{j k}(\boldsymbol{\theta})}{\partial \theta_{u} \partial \theta_{v}}\right]$.

The $(u, v)$ th element of the item information matrix associated with ranking $k$ can be derived as follows:

$$
\begin{gathered}
{\left[I_{j k}(\theta) P_{j k}(\theta)\right]_{u, v}=-\frac{\partial^{2} \ln P_{j k}(\theta)}{\partial \theta_{u} \partial \theta_{v}} P_{j k}(\theta)=\left[-\frac{\partial}{\partial \theta_{v}}\left(\frac{1}{P_{j k}(\theta)} \frac{\partial P_{j k}(\theta)}{\partial \theta_{u}}\right)\right] P_{j k}(\theta)} \\
=\frac{\left(\frac{\partial P_{j k}(\theta)}{\partial \theta_{u}}\right)\left(\frac{\partial P_{j k}(\theta)}{\partial \theta_{v}}\right)-\left(\frac{\partial^{2} P_{j k}(\theta)}{\partial \theta_{u} \partial \theta_{v}}\right) P_{j k}(\theta)}{\left[P_{j k}(\theta)\right]^{2}} P_{j k}(\theta) \\
=\frac{\left(\frac{\partial P_{j k}(\theta)}{\partial \theta_{u}}\right)\left(\frac{\partial P_{j k}(\theta)}{\partial \theta_{v}}\right)}{P_{j k}(\theta)}-\left(\frac{\partial^{2} P_{j k}(\theta)}{\partial \theta_{u} \partial \theta_{v}}\right) .
\end{gathered}
$$

With matrix notation, Eq. 11 becomes a $D \times D$ information matrix:

$I_{j}(\theta)=\sum_{k=1}^{m_{j}} \frac{\left[\frac{\partial P_{j k}(\theta)}{\partial \theta}\right]\left[\frac{\partial P_{j k}(\theta)}{\partial \theta}\right]^{T}}{P_{j k}(\theta)}-\frac{\partial^{2} P_{j k}(\theta)}{\partial \theta \partial \theta^{T}}$,

where $P_{j k}(\boldsymbol{\theta})$ is the GGUM-RANK probability of a respondent providing a particular ranking $k\left(k=1,2, \ldots, m_{j}\right)$ for MFC item $j$, given $\boldsymbol{\theta} ; m_{j}$ is the total number of possible rankings, $\frac{\partial P_{j k}(\boldsymbol{\theta})}{\partial \boldsymbol{\theta}}$ is a $D \times 1$ gradient vector, and $\frac{\partial^{2} P_{j k}(\boldsymbol{\theta})}{\partial \boldsymbol{\theta} \partial \boldsymbol{\theta}^{T}}$ is a $D \times D$ Hessian matrix. For MFC pair, triplet, and tetrad items, $m_{j}$ equals $2 !=2,3 !=6$, and $4 !=24$, respectively. The gradient vector in Eq. 15, given by the first-order partial derivative of the GGUM-RANK probability function with respect to $\theta$, is

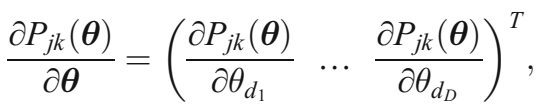

and the numerator of the first term in Eq. 15 becomes

$\left[\frac{\partial P_{j k}(\boldsymbol{\theta})}{\partial \boldsymbol{\theta}}\right]\left[\frac{\partial P_{j k}(\boldsymbol{\theta})}{\partial \boldsymbol{\theta}}\right]^{T}=\left(\begin{array}{ccc}{\left[\frac{\partial P_{j k}(\boldsymbol{\theta})}{\partial \theta_{d_{1}}}\right]^{2}} & \ldots & {\left[\frac{\partial P_{j k}(\boldsymbol{\theta})}{\partial \theta_{d_{1}}}\right]} \\ \vdots & \ddots & {\left[\frac{\partial P_{j k}(\boldsymbol{\theta})}{\partial \theta_{d_{D}}}\right]} \\ {\left[\frac{\partial P_{j k}(\boldsymbol{\theta})}{\partial \theta_{d_{D}}}\right]\left[\frac{\partial P_{j k}(\boldsymbol{\theta})}{\partial \theta_{d_{1}}}\right]} & \cdots & {\left[\frac{\partial P_{j k}(\boldsymbol{\theta})}{\partial \theta_{d_{D}}}\right]^{2}}\end{array}\right)$.

For the second term in Eq. 15, the Hessian matrix can be formed by taking the second-order partial derivative of the GGUM-RANK probability function with respect to $\boldsymbol{\theta}^{T}=$ $\left(\theta_{d_{1}}, \theta_{d_{2}}, \ldots, \theta_{d_{D}}\right)$, as follows:

$\frac{\partial^{2} P_{j k}(\boldsymbol{\theta})}{\partial \boldsymbol{\theta} \partial \boldsymbol{\theta}^{T}}=\frac{\partial}{\partial \boldsymbol{\theta}^{T}}\left(\begin{array}{lll}\frac{\partial P_{j k}(\boldsymbol{\theta})}{\partial \theta_{d_{1}}} & \ldots & \frac{\partial P_{j k}(\boldsymbol{\theta})}{\partial \theta_{d_{D}}}\end{array}\right)^{T}=\left(\begin{array}{ccc}\frac{\partial^{2} P_{j k}(\boldsymbol{\theta})}{\partial \theta_{d_{1}}^{2}} & \ldots & \frac{\partial^{2} P_{j k}(\boldsymbol{\theta})}{\partial \theta_{d_{1}} \partial \theta_{d_{D}}} \\ \vdots & \ddots & \vdots \\ \frac{\partial^{2} P_{j k}(\boldsymbol{\theta})}{\partial \theta_{d_{D}} \partial \theta_{d_{1}}} & \ldots & \frac{\partial^{2} P_{j k}(\boldsymbol{\theta})}{\partial \theta_{d_{D}}^{2}}\end{array}\right)$.
We, thus, have the general formulation of the item information matrix, $I_{j}(\boldsymbol{\theta})$. The test information matrix is obtained by summing the item information matrices over all items, $I(\boldsymbol{\theta})$

$=\sum_{j} I_{j}(\boldsymbol{\theta})$, and asymptotic standard errors are given by the reciprocal square root of the diagonal elements.

For illustration, Fig. 1 presents a two-dimensional GGUMRANK item response function (IRF) and the corresponding item information function (IIF) for an MFC pair composed of statements $\mathrm{A}$ and $\mathrm{B}$, having parameters $\left(\alpha_{A}=1.5, \delta_{A}=2.0\right.$, $\left.\tau_{A}=-1.0\right)$ and $\left(\alpha_{B}=1.5, \delta_{B}=-2.0, \tau_{B}=-1.0\right)$. The IRF shows the probability of choosing statement A over statement B or, equivalently, the probability of the ranking $\mathrm{A}>\mathrm{B}$, at different $\left(\theta_{d_{A}}, \theta_{d_{B}}\right)$. Note that item information is lowest where $\theta=\delta$ on the respective dimensions, as with unidimensional singlestatement ideal point models (Joo, Chun, Stark, \& Chernyshenko, 2019; Roberts et al., 2000; Stark et al., 2005).

The multidimensional item information functions for the GGUM-RANK model were computed on the basis of our derivation (Eq. 15), and the first- and second-order partial derivatives (Eqs. 16-18) were obtained from numerical approximations provided by a separate Ox (Doornik, 2009) program. The accuracy of the numerical approximation was checked by comparing the GGUM-RANK pair results with analytic results based on the equations in Stark et al. (2005). ${ }^{1}$ Note that the numerical approximation method is consistent with the previous GGUM-RANK study (Joo et al., 2018).

\section{Latent trait estimation}

To estimate latent trait parameters for the GGUM-RANK model, expected a posteriori (EAP; Bock \& Mislevy, 1982) estimation can be used. In multidimensional EAP estimation, a vector of latent trait estimates is obtained for each respondent by taking the mean of the posterior density of $\boldsymbol{\theta}$. The EAP estimate $\hat{\boldsymbol{\theta}}_{E A P}$ and its posterior covariance matrix $\operatorname{Cov}(\boldsymbol{\theta} \mid \boldsymbol{X})$ are given by

$\hat{\boldsymbol{\theta}}_{E A P}=E(\boldsymbol{\theta} \mid \boldsymbol{X})=\frac{\int \ldots \int \theta L(\boldsymbol{X} \mid \boldsymbol{\theta}) f(\boldsymbol{\theta}) d \boldsymbol{\theta}}{\int \ldots \int L(\boldsymbol{X} \mid \boldsymbol{\theta}) f(\boldsymbol{\theta}) d \boldsymbol{\theta}}$,

$\operatorname{Cov}(\boldsymbol{\theta} \mid \boldsymbol{X})=\frac{\int \ldots \int\left(\boldsymbol{\theta}-\hat{\boldsymbol{\theta}}_{E A P}\right)\left(\boldsymbol{\theta}-\hat{\boldsymbol{\theta}}_{E A P}\right)^{T} L(\boldsymbol{X} \mid \boldsymbol{\theta}) f(\boldsymbol{\theta}) d \boldsymbol{\theta}}{\int \ldots \int L(\boldsymbol{X} \mid \boldsymbol{\theta}) f(\boldsymbol{\theta}) d \boldsymbol{\theta}}$

where $\mathbf{X}$ is a matrix of item responses, $L(\boldsymbol{X} \mid \boldsymbol{\theta})$ is the likelihood of the response data across items, based on the GGUMRANK model, and $f(\boldsymbol{\theta})$ is a prior distribution for $\boldsymbol{\theta}$.

For our study comparing latent trait estimation with fixedlength nonadaptive and adaptive GGUM-RANK pair, triplet, and tetrad tests, which is described shortly, a multivariate standard normal probability density function was used for $f(\boldsymbol{\theta})$, in

\footnotetext{
${ }^{1}$ The first- and second-order partial derivatives for the GGUM-RANK triplet are available in the Appendix of Joo et al. (2018)
} 
a

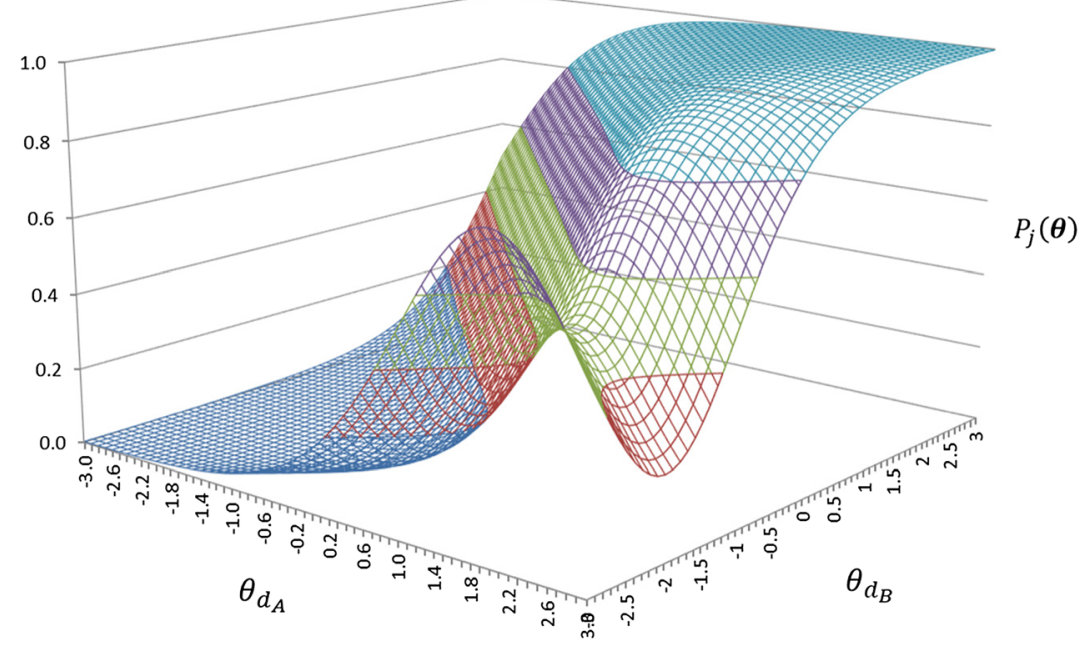

b

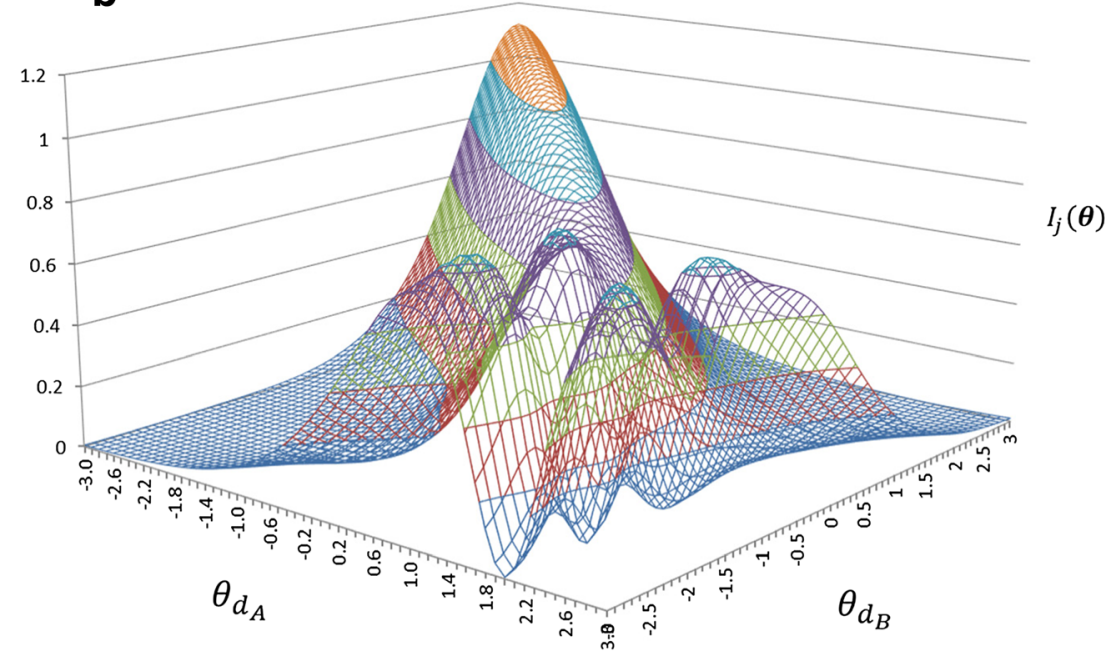

Fig. 1 GGUM-RANK item response function (a) and item information function (b) for the MFC pair with statement parameters $\left(\alpha_{A}=1.5, \delta_{A}=2.0, \tau_{A}=-\right.$ $1.0)$ and $\left(\alpha_{B}=1.5, \delta_{B}=-2.0, \tau_{B}=-1.0\right)$

accordance with previous research (e.g., de la Torre et al., 2012; Hontangas et al., 2015). The multidimensional integrations in Eqs. 19 and 20 were accomplished using Gaussian integration with ten evenly spaced quadrature nodes ranging from -3 to +3 on each dimension, ${ }^{2}$ and the standard errors of the EAP estimates (i.e., posterior standard deviation [PSD]) were obtained by taking the square root of the diagonal elements of $\operatorname{Cov}(\theta \mid X)$.

\section{CAT implementation}

Suppose we have a large pool of MFC items, each involving $D$ dimensions, where $D=2$ for pairs, $D=3$ for triplets, and $D=$

\footnotetext{
${ }^{2}$ We conducted a preliminary study by increasing the number of quadrature nodes to 20 and 30 per dimension and found that the EAP estimation did not improve significantly. Note that this finding is consistent with the previous study with GGUM-RANK EAP estimation (Hontangas et al., 2015).
}

4 for tetrads. We developed a GGUM-RANK CAT algorithm that operated as follows:

1. Initialize the vector of latent trait estimates to 0 for all dimensions - that is, let $\hat{\boldsymbol{\theta}}_{j=0}=\mathbf{0}$.

2. Compute GGUM-RANK response probabilities and item information matrices for all items in the pool at the initial latent trait values.

3. Select the item for which the trace of the item information matrix is maximum.

4. Simulate an MFC rank response (details provided later).

5. Estimate the respondent's latent trait scores using EAP estimation and update the vector of latent trait estimates, $\hat{\boldsymbol{\theta}}_{j}$.

6. Remove the selected MFC item from the pool, and repeat Steps $2-5$ until the desired number of items $j=1,2, \ldots, J$ has been administered. 
7. Estimate the final latent trait parameters as $\hat{\boldsymbol{\theta}}_{J}$ and their standard errors $\operatorname{PSD}\left(\hat{\boldsymbol{\theta}}_{J}\right)$.

It is worthwhile to note that a previous study explored three methods of summarizing the information matrix for item selection in multidimensional adaptive testing (Mulder \& van der Linden, 2009): A-optimality (trace), D-optimality (determinant), and E-optimality (eigenvalue). We chose the trace method for the GGUM-RANK model because (1) it provided results consistent with the previous MFC studies' definition of information for the GGUM-RANK model (e.g., Joo et al., 2018); (2) it is the consistent approach for the CAT algorithm with the MUPP model, which is equivalent to GGUM-RANK pair (Stark et al., 2005); and (3) the trace method is comparable to the determinant method, and better than the eigenvalue approach.

\section{Method}

\section{Study design}

A simulation study was conducted to investigate GGUMRANK latent trait estimation using a design similar to that of Stark et al. (2012) for comparing nonadaptive and adaptive tests. In addition to manipulating the item selection method (nonadaptive, CAT) and MFC format (pair, triplet, tetrad), we varied the true correlations between dimensions $(0, .3, .5)$, the number of items administered $(5,10,20)$, and the percentage of items that were required to be unidimensional $(0 \%, 20 \%)$. Dimensionality was fixed at $D=2$, $D=3$, and $D=4$ for the pair, triplet, and tetrad tests, respectively. Note that a nonzero percentage of unidimensional items was considered because Stark et al. (2005) suggested that this might improve scoring accuracy. For the CAT algorithm, unidimensional items were fixed prior to the item selection for the multidimensional items, so that the percentage of unidimensional items was guaranteed. When five items were administered, for example, one item was fixed and administered as unidimensional, and the other four items were selected from the item pool, which included all multidimensional items. ${ }^{3}$

For each format, a pool of 100 MFC items was randomly generated. Both nonadaptive and adaptive tests were required to satisfy basic "content" constraints. For example, in the triplet $0 \%$ unidimensional conditions, all administered items were " $1-2-3$ " combinations, meaning that statement A represented Dimension 1, B represented Dimension 2, and $\mathrm{C}$ represented Dimension 3; whereas in

\footnotetext{
${ }^{3}$ Note that unidimensional items were fixed in this study, but the unidimensional items can also be adaptive from the unidimensional item pool.
}

the triplet $20 \%$ unidimensional conditions, $20 \%$ of the administered items were $1-1-1,2-2-2$, or $3-3-3$ combinations (with precautions taken to balance the numbers), and the rest were 1-2-3 combinations. Beyond that, items were selected randomly from the respective pools in the nonadaptive test conditions, on the basis of maximum information in the CAT conditions.

In total, there were 2 (item selection method) $\times 3$ (MFC format) $\times 3$ (theta correlation) $\times 3$ (number of administered items $) \times 2$ (percentage of unidimensional items $)=108$ simulation conditions, with 1,000 respondents in each condition. For each condition, 100 replications were performed. All of the simulation code was written in the Ox programming language (Doornik, 2009).

\section{Data generation and trait estimation}

For each respondent, a $D$-dimensional vector for generating latent trait scores of appropriate dimensionality was randomly sampled from a multivariate normal distribution with a zero mean vector and a correlation structure specific to the simulation condition (i.e., $0, .3, .5$ ). The statement parameters were also randomly generated for both nonadaptive and adaptive tests. More specifically, GGUM discrimination parameters $(\alpha)$ and location parameters $(\delta)$ were randomly sampled from a uniform distribution $\mathrm{U}(.5,2)$ and a normal distribution $\mathrm{N}(0$, $1)$, respectively, and the threshold parameters $(\tau)$ were fixed at -1 across conditions. ${ }^{4}$ These statement parameters were generated as in previous GGUM estimation studies (e.g., de la Torre, Stark, \& Chernyshenko, 2006; Hontangas et al., 2015; Joo, Lee, \& Stark, 2017).

Using the randomly generated vectors of latent trait scores and statement parameters for MFC items, GGUM-RANK probabilities were computed for each possible ranking and used to demark segments of a cumulative probability continuum. A random number between 0 and 1 was then sampled from a uniform distribution, and the numerical code for the ranking was assigned corresponding to the segment containing that random number. For each data type, each possible ranking was assigned a sequential numerical code starting with 1 ; that is, the possible rankings were coded $1-2$ for pairs, 1-6 for triplets, and 1-24 for tetrads.

\section{Analyses}

The simulation study results were evaluated using several criteria: average bias (BIAS), root mean squared error (RMSE), correlation between the generating and estimated

\footnotetext{
${ }^{4}$ We fixed tau parameters in this study following Hontangas et al. (2015). They fixed tau parameters in order to show the impact of discrimination and location parameters for GGUM-RANK scoring. Note that this constraint can be relaxed.
} 
thetas (CORR), and the posterior standard deviation of EAP estimates (PSD). BIAS and RMSE were computed as follows:

$\mathrm{BIAS}=\sum_{n=1}^{N} \frac{\hat{\theta}_{n}-\theta_{n}}{N}$,

$\mathrm{RMSE}=\sqrt{\sum_{n=1}^{N} \frac{\left(\hat{\theta}_{n}-\theta_{n}\right)^{2}}{N}}$,

where $\hat{\theta}_{n}$ is the estimated parameter, $\theta_{n}$ is the generating parameter, and $N$ is the number of respondents. In addition, to compare the reliability of the various MFC measures, marginal reliability (REL) was computed (Brown \& Croudace, 2015):

$\bar{\rho}=1-\frac{\bar{\sigma}_{e}^{2}}{\sigma_{\hat{\theta}}^{2}}$

where $\sigma_{\widehat{\theta}}^{2}=\frac{1}{N} \sum_{n=1}^{N}\left(\widehat{\theta_{n}}-\widehat{\widehat{\theta}_{n}}\right)^{2}$ is the variance of the latent trait estimates, and $\bar{\sigma}_{e}^{2}=\frac{1}{N} \sum_{n=1}^{N} P S D^{2}\left(\hat{\theta}_{n}\right)$ is the average squared standard error. BIAS, RMSE, PSD, CORR, and REL statistics were computed for each dimension and then averaged across dimensions for summary reporting.

\section{Results}

\section{Adaptive and nonadaptive tests}

Tables 1, 2, and 3 present the latent trait estimation results for the conditions in which correlation between dimensions were $0, .3$, and .5 , respectively. Consistent with expectations, CATs performed substantially better than similarly configured nonadaptive tests. For example, PSDs of GGUM-RANK pairs for nonadaptive tests ranged from .71 to .37 versus .62 to .28 for adaptive tests. Similarly, CORRs for nonadaptive tests ranged from .71 to 96 as opposed to .76 to .97 for adaptive tests across simulation conditions. The similar pattern was consistently observed for BIAS, RMSE, and REL across conditions as well. Note that the PSD and CORR values for GGUM-RANK pairs were comparable to results in the MUPP CAT study (Stark et al., 2012) for similar test length and dimensionality. In accordance with Stark et al. (2012), correlations between estimated and known trait scores (i.e., CORR) ranged from .69 to .92 and .82 to .93 for nonadaptive and adaptive tests, respectively (see Table 4 in Stark et al., 2012).

Note that the outperformance of CATs was consistent across the pair, triplet, and tetrad formats, but has been explicitly examined only in GGUM-RANK pairs. In addition, with a small number of items, GGUM-RANK CAT provided more efficient latent trait estimates than nonadaptive tests. For instance, in the condition in which dimensions were uncorrelated and the percentage of unidimensional items was zero, CORRs of nonadaptive and adaptive tests were .72 and .80 for GGUMRANK pairs with five items, whereas the corresponding values were .95 and .96 for GGUM-RANK tetrads with 20 items.

It is worthwhile to note that, in the present study, latent traits were estimated accurately overall for both the nonadaptive and adaptive tests. As is shown in Tables 1, 2 and 3 , BIAS ranged from -.06 to .07 for nonadaptive tests and from - .08 to .04 for adaptive tests across conditions. The BIAS values can be considered small, given that an estimate with bias less than .05 is generally known as an unbiased estimate in simulation studies (Hoogland \& Boomsma, 1998).

\section{MFC formats}

Consistent with the previous studies (Joo et al., 2018; P. Lee, Joo, Stark, \& Chernyshenko, 2019), GGUM-RANK triplet and tetrad formats provided better results than pairs with both nonadaptive and adaptive item selection. For example, using the values in Table 1, the average RMSE for pair tests was .51 , versus .41 and .41 for triplet and tetrad tests, respectively. Similarly, the average CORR for pair tests was .85 , as opposed to .89 and .90 for triplet and tetrad tests. Note that although tetrads generally showed better performance than triplets, somewhat inconsistent values were found (e.g., triplet RMSEs were smaller than tetrad RMSEs). This might have been due to sampling errors in the data generation and the relatively small number of replications. Note that the RMSE, PSD, CORR, and REL values for nonadaptive tests were comparable to those in the previous study that compared latent trait estimation for GGUM-RANK pairs, triplets, and tetrads (Joo et al., 2018).

More importantly, we found that GGUM-RANK CAT pairs performed similarly to or better than nonadaptive triplets for the same test length. Furthermore, GGUMRANK CAT triplets also performed similarly to or better than nonadaptive tetrads. For example, in the conditions in which the dimension correlation was .5 (see Table 3), the CORRs for GGUM-RANK CAT pairs ranged from .81 to .97 , versus .75 to .96 for nonadaptive triplets. Similarly, in the same condition, the CORRs for GGUM-RANK CAT triplets ranged from .90 to .97 , as opposed to .83 to .95 for nonadaptive tetrads. The same pattern was consistently observed from RMSE, PSD, and REL, as well. 
Table 1 Latent trait estimation results for GGUM-RANK nonadaptive and adaptive tests when dimensions were uncorrelated

\begin{tabular}{|c|c|c|c|c|c|c|c|c|c|c|c|c|}
\hline \multirow[t]{2}{*}{ Format } & \multirow[t]{2}{*}{$\%$ Uni } & \multirow[t]{2}{*}{ Items } & \multicolumn{2}{|l|}{ BIAS } & \multicolumn{2}{|c|}{ RMSE } & \multicolumn{2}{|l|}{ PSD } & \multicolumn{2}{|c|}{ CORR } & \multicolumn{2}{|l|}{ REL } \\
\hline & & & $\mathrm{NON}$ & CAT & $\mathrm{NON}$ & CAT & $\mathrm{NON}$ & CAT & $\mathrm{NON}$ & CAT & $\mathrm{NON}$ & CAT \\
\hline \multirow[t]{6}{*}{ Pair } & \multirow[t]{3}{*}{0} & 5 & .054 & .042 & .715 & .656 & .710 & .614 & .717 & .800 & .293 & .341 \\
\hline & & 10 & -.048 & -.021 & .566 & .473 & .553 & .423 & .841 & .891 & .560 & .718 \\
\hline & & 20 & .026 & .034 & .375 & .250 & .368 & .278 & .910 & .971 & .799 & .915 \\
\hline & \multirow[t]{3}{*}{20} & 5 & -.061 & -.079 & .745 & .632 & .695 & .621 & .711 & .762 & .397 & .490 \\
\hline & & 10 & .028 & -.016 & .601 & .408 & .538 & .407 & .829 & .910 & .487 & .771 \\
\hline & & 20 & -.005 & -.012 & .442 & .291 & .401 & .277 & .887 & .957 & .764 & .901 \\
\hline \multirow[t]{6}{*}{ Triplet } & \multirow[t]{3}{*}{0} & 5 & .058 & .020 & .659 & .460 & .617 & .484 & .747 & .886 & .440 & .649 \\
\hline & & 10 & -.024 & -.017 & .458 & .382 & .445 & .331 & .864 & .919 & .704 & .838 \\
\hline & & 20 & .046 & .009 & .311 & .229 & .285 & .238 & .950 & .973 & .893 & .928 \\
\hline & \multirow[t]{3}{*}{20} & 5 & .006 & -.006 & .603 & .482 & .606 & .480 & .780 & .882 & .466 & .629 \\
\hline & & 10 & .020 & -.001 & .501 & .331 & .467 & .334 & .857 & .938 & .725 & .825 \\
\hline & & 20 & .006 & .007 & .295 & .225 & .296 & .241 & .952 & .974 & .885 & .913 \\
\hline \multirow[t]{6}{*}{ Tetrad } & \multirow[t]{3}{*}{0} & 5 & .029 & .006 & .570 & .567 & .536 & .467 & .823 & .846 & .528 & .644 \\
\hline & & 10 & .000 & .003 & .465 & .343 & .370 & .339 & .887 & .939 & .817 & .847 \\
\hline & & 20 & -.004 & .019 & .304 & .269 & .248 & .295 & .950 & .962 & .910 & .942 \\
\hline & \multirow[t]{3}{*}{20} & 5 & -.036 & -.008 & .567 & .508 & .530 & .444 & .821 & .859 & .556 & .676 \\
\hline & & 10 & .013 & -.008 & .434 & .315 & .386 & .329 & .900 & .942 & .762 & .838 \\
\hline & & 20 & .035 & -.016 & .324 & .278 & .229 & .230 & .947 & .956 & .935 & .931 \\
\hline
\end{tabular}

$\%$ Uni $=$ percentage of total items that were unidimensional, Items $=$ number of administered MFC items, NON $=$ nonadaptive test, CAT $=$ adaptive test, $\mathrm{BIAS}=$ average bias, $\mathrm{RMSE}=$ root mean square error, $\mathrm{PSD}=$ posterior standard deviation, $\mathrm{CORR}=$ correlation between generated and estimated parameters, $\mathrm{REL}=$ marginal reliability

\section{Percent of unidimensional items and dimension correlations}

On the basis of the study results, it was evident that GGUMRANK CATs outperformed nonadaptive tests under the conditions in which dimension correlation was low or high and the percentage of unidimensional items was zero or .20.
However, no substantial differences were seen in connection with the percentage of unidimensional items and different levels of correlation between latent trait dimensions. Note that these patterns are consistent with the previous studies on MUPP CAT (Stark et al., 2012) and nonadaptive GGUMRANK modeling (Joo et al., 2018; P. Lee, Joo, Stark, \& Chernyshenko, 2019). As expected, increasing the number

Table 2 Latent trait estimation results for GGUM-RANK nonadaptive and adaptive tests when dimensions were correlated (.3)

\begin{tabular}{|c|c|c|c|c|c|c|c|c|c|c|c|c|}
\hline \multirow[t]{2}{*}{ Format } & \multirow[t]{2}{*}{$\%$ Uni } & \multirow[t]{2}{*}{ Items } & \multicolumn{2}{|l|}{ BIAS } & \multicolumn{2}{|c|}{ RMSE } & \multicolumn{2}{|l|}{ PSD } & \multicolumn{2}{|c|}{ CORR } & \multicolumn{2}{|l|}{ REL } \\
\hline & & & $\mathrm{NON}$ & CAT & $\mathrm{NON}$ & CAT & $\mathrm{NON}$ & CAT & NON & CAT & $\mathrm{NON}$ & CAT \\
\hline \multirow[t]{6}{*}{ Pair } & \multirow[t]{3}{*}{0} & 5 & .046 & -.050 & .671 & .566 & 690 & .571 & .711 & .837 & .262 & .329 \\
\hline & & 10 & -.020 & -.039 & .613 & .381 & .549 & .397 & .773 & .933 & .493 & .798 \\
\hline & & 20 & .020 & .007 & .344 & .277 & .374 & .300 & .934 & .973 & .825 & .946 \\
\hline & \multirow[t]{3}{*}{20} & 5 & .001 & -.005 & .688 & .565 & 699 & .594 & .772 & .795 & .396 & .468 \\
\hline & & 10 & -.011 & -.032 & .570 & .498 & .528 & .404 & .833 & .897 & .595 & .807 \\
\hline & & 20 & .053 & -.030 & .421 & .262 & .389 & .272 & .905 & .967 & .800 & .910 \\
\hline \multirow[t]{6}{*}{ Triplet } & \multirow[t]{3}{*}{0} & 5 & -.022 & -.002 & .596 & .502 & .601 & .459 & .764 & .849 & .491 & .663 \\
\hline & & 10 & -.029 & .016 & .464 & .316 & .427 & .329 & .866 & .947 & .705 & .852 \\
\hline & & 20 & .000 & -.013 & .319 & .240 & .289 & .235 & .954 & .967 & .911 & .931 \\
\hline & \multirow[t]{3}{*}{20} & 5 & .004 & -.043 & .621 & .495 & .620 & .469 & .809 & .891 & .465 & .712 \\
\hline & & 10 & -.029 & -.009 & .467 & .314 & .468 & .318 & .880 & .944 & .689 & .850 \\
\hline & & 20 & -.002 & .002 & .329 & .249 & .288 & .236 & .937 & .968 & .895 & .925 \\
\hline \multirow[t]{6}{*}{ Tetrad } & \multirow[t]{3}{*}{0} & 5 & -.006 & .023 & .523 & .505 & .515 & .435 & .825 & .874 & .584 & .723 \\
\hline & & 10 & -.014 & -.034 & .396 & .360 & .376 & .317 & .927 & .930 & .822 & .845 \\
\hline & & 20 & .010 & .018 & .286 & .275 & .235 & .280 & .953 & .963 & .918 & .932 \\
\hline & \multirow[t]{3}{*}{20} & 5 & -.018 & .003 & .556 & .442 & .528 & .422 & .819 & .880 & .585 & .722 \\
\hline & & 10 & -.019 & -.002 & .388 & .305 & .375 & .317 & .925 & .947 & .814 & .854 \\
\hline & & 20 & -.008 & -.007 & .326 & .241 & .245 & .238 & .950 & .967 & .925 & .936 \\
\hline
\end{tabular}

$\%$ Uni $=$ percentage of total items that were unidimensional, Items $=$ number of administered MFC items, NON = nonadaptive test, CAT $=$ adaptive test, BIAS $=$ average bias, $\mathrm{RMSE}=$ root mean square error, $\mathrm{PSD}=$ posterior standard deviation, $\mathrm{CORR}=$ correlation between generated and estimated parameters, $\mathrm{REL}=$ marginal reliability 
Table 3 Latent trait estimation results for GGUM-RANK nonadaptive and adaptive tests when dimensions were correlated (.5)

\begin{tabular}{|c|c|c|c|c|c|c|c|c|c|c|c|c|}
\hline \multirow[t]{2}{*}{ Format } & \multirow[t]{2}{*}{$\%$ Uni } & \multirow[t]{2}{*}{ Items } & \multicolumn{2}{|l|}{ BIAS } & \multicolumn{2}{|c|}{ RMSE } & \multicolumn{2}{|l|}{ PSD } & \multicolumn{2}{|c|}{ CORR } & \multicolumn{2}{|l|}{ REL } \\
\hline & & & $\mathrm{NON}$ & CAT & $\mathrm{NON}$ & CAT & $\mathrm{NON}$ & CAT & $\mathrm{NON}$ & CAT & $\mathrm{NON}$ & CAT \\
\hline \multirow[t]{6}{*}{ Pair } & \multirow[t]{3}{*}{0} & 5 & .058 & -.033 & .628 & .608 & .665 & .551 & .725 & .806 & .213 & .543 \\
\hline & & 10 & -.029 & .017 & .539 & .375 & .523 & .379 & .856 & .927 & .632 & .818 \\
\hline & & 20 & .000 & .020 & .347 & .266 & .388 & .263 & .928 & .952 & .756 & .897 \\
\hline & \multirow[t]{3}{*}{20} & 5 & -.013 & .030 & .617 & .583 & .664 & .555 & .759 & .804 & .411 & .500 \\
\hline & & 10 & .071 & -.048 & .570 & .385 & .529 & .420 & .840 & .916 & .625 & .767 \\
\hline & & 20 & .024 & -.004 & .387 & .258 & .403 & .282 & .926 & .968 & .805 & .898 \\
\hline \multirow[t]{6}{*}{ Triplet } & \multirow[t]{3}{*}{0} & 5 & .012 & .011 & .712 & .416 & .598 & .442 & .751 & .899 & .427 & .707 \\
\hline & & 10 & -.011 & .004 & .458 & .359 & .415 & .318 & .884 & .939 & .764 & .883 \\
\hline & & 20 & -.016 & -.007 & .278 & .245 & .288 & .244 & .952 & .973 & .889 & .929 \\
\hline & \multirow[t]{3}{*}{20} & 5 & -.019 & .002 & .595 & .448 & .579 & .451 & .802 & .900 & .490 & .730 \\
\hline & & 10 & .029 & .025 & .444 & .324 & .443 & .314 & .884 & .953 & .731 & .888 \\
\hline & & 20 & .021 & -.011 & .307 & .225 & .289 & .238 & .955 & .972 & .910 & .927 \\
\hline \multirow[t]{6}{*}{ Tetrad } & \multirow[t]{3}{*}{0} & 5 & -.009 & .025 & .540 & .415 & .496 & .394 & .834 & .896 & .628 & .754 \\
\hline & & 10 & -.012 & .020 & .377 & .320 & .360 & .307 & .911 & .943 & .795 & .864 \\
\hline & & 20 & -.018 & .009 & .305 & .253 & .244 & .259 & .948 & .967 & .915 & .936 \\
\hline & \multirow[t]{3}{*}{20} & 5 & -.013 & -.029 & .534 & .429 & .508 & .412 & .857 & .895 & .571 & .780 \\
\hline & & 10 & .003 & -.004 & .388 & .317 & .364 & .303 & .913 & .934 & .812 & .852 \\
\hline & & 20 & -.008 & .002 & .321 & .224 & .254 & .247 & .955 & .972 & .933 & .935 \\
\hline
\end{tabular}

$\%$ Uni $=$ percentage of total items that were unidimensional, Items $=$ number of administered MFC items, NON $=$ nonadaptive test, CAT $=$ adaptive test, $\mathrm{BIAS}=$ average bias, $\mathrm{RMSE}=$ root mean square error, $\mathrm{PSD}=$ posterior standard deviation, $\mathrm{CORR}=$ correlation between generated and estimated parameters, $\mathrm{REL}=$ marginal reliability

of items substantially increased the accuracy of the latent trait estimates.

\section{Follow-up study}

MFC tests have often been constructed with higher dimensions in applied settings (e.g., TAPAS; Drasgow et al., 2012;
Stark et al., 2014). Following a reviewer's suggestion, we conducted a follow-up study to examine the performance of the methods in more practical situations involving a higher number of dimensions.

For this purpose, we extended the number of dimensions to ten (i.e., $D=10$ ) and compared the performance of CAT with nonadaptive tests. We also compared the scoring accuracy of

Table 4 Latent trait estimation results for ten-dimension GGUM-RANK nonadaptive and adaptive tests

\begin{tabular}{|c|c|c|c|c|c|c|c|c|c|c|c|c|}
\hline & & & BIAS & & RMSE & & PSD & & CORR & & REL & \\
\hline Corr & Format & Items & NON & CAT & $\mathrm{NON}$ & CAT & $\mathrm{NON}$ & CAT & $\mathrm{NON}$ & CAT & $\mathrm{NON}$ & CAT \\
\hline \multirow[t]{9}{*}{0} & \multirow[t]{3}{*}{ Pair } & 30 & -.004 & .004 & .634 & .581 & .624 & .571 & .773 & .791 & .440 & .462 \\
\hline & & 60 & .044 & -.031 & .529 & .490 & .519 & .464 & .841 & .876 & .700 & .766 \\
\hline & & 90 & .012 & -.008 & .465 & .405 & .463 & .385 & .889 & .916 & .802 & .837 \\
\hline & \multirow[t]{3}{*}{ Triplet } & 30 & -.021 & .007 & .533 & .466 & .497 & .437 & .840 & .887 & .819 & .842 \\
\hline & & 60 & -.019 & .008 & .492 & .433 & .450 & .387 & .874 & .905 & .847 & .882 \\
\hline & & 90 & .014 & -.010 & .338 & .301 & .321 & .283 & .921 & .941 & .902 & .925 \\
\hline & \multirow[t]{3}{*}{ Tetrad } & 30 & .019 & .019 & .525 & .490 & .504 & .458 & .858 & .913 & .862 & .877 \\
\hline & & 60 & .000 & .000 & .479 & .406 & .447 & .394 & .905 & .952 & .866 & .896 \\
\hline & & 90 & -.025 & .019 & .344 & .244 & .325 & .238 & .957 & .967 & .910 & .930 \\
\hline \multirow[t]{9}{*}{.5} & \multirow[t]{3}{*}{ Pair } & 30 & .001 & .014 & .691 & .595 & .641 & .578 & .713 & .742 & .443 & .461 \\
\hline & & 60 & .009 & -.029 & .550 & .517 & .522 & .503 & .814 & .831 & .687 & .753 \\
\hline & & 90 & .065 & -.003 & .467 & .448 & .464 & .408 & .835 & .862 & .800 & .836 \\
\hline & \multirow[t]{3}{*}{ Triplet } & 30 & -.007 & -.020 & .542 & .482 & .466 & .466 & .828 & .868 & .815 & .826 \\
\hline & & 60 & .012 & .000 & .504 & .438 & .471 & .424 & .860 & .895 & .843 & .856 \\
\hline & & 90 & .001 & -.004 & .336 & .296 & .315 & .291 & .913 & .946 & .889 & .922 \\
\hline & \multirow[t]{3}{*}{ Tetrad } & 30 & .035 & .002 & .501 & .481 & .448 & .419 & .854 & .900 & .853 & .888 \\
\hline & & 60 & -.005 & -.012 & .470 & .386 & .441 & .369 & .894 & .927 & .862 & .906 \\
\hline & & 90 & .001 & .021 & .359 & .265 & .318 & .253 & .952 & .957 & .897 & .937 \\
\hline
\end{tabular}

Corr $=$ Dimension correlations, Items $=$ number of administered MFC items, NON = nonadaptive test, $\mathrm{CAT}=$ adaptive test, BIAS $=$ average bias, RMSE $=$ root mean square error, $\mathrm{PSD}=$ posterior standard deviation, $\mathrm{CORR}=$ correlation between generated and estimated parameters, $\mathrm{REL}=$ marginal reliability. Total number of items in the item bank $=300$ 
MFC pairs, triplets, and tetrads for both CAT and nonadaptive tests. Because the number of dimensions was increased, we also increased the numbers of items administered to 30,60 , and 90. We followed P. Lee, Joo, Stark, and Chernyshenko (2019) in order to create well-balanced pair, triplet, and tetrad MFC tests with ten dimensions. Readers are referred to the online appendix in P. Lee, Joo, Stark, and Chernyshenko for detailed information about the dimension specifications. To show the benefits of MFC CAT in higher-dimensionality tests, we increased the number of items in the item bank to 300 . Finally, we varied the correlations between dimensions to 0 and .5 in the follow-up study. The percentage of unidimensional items was not considered, because no distinct pattern was found in our main simulation study.

Table 4 shows the EAP estimation results from the followup study. ${ }^{5}$ To be consistent with the main study result reporting, the evaluation criteria (e.g., BIAS, RMSE, PSD, CORR, and REL) were computed for ten dimensions and then averaged across dimensions. Overall, the results in the tendimension conditions were similar to those in the lowerdimensionality conditions. MFC CATs consistently outperformed nonadaptive tests, and the values were similar to the previous study results. For example, in the tendimension condition, the averaged CORR across nonadaptive triplet tests was .87 , as opposed to .91 for triplet CAT. In addition, the triplet results were comparable to those from $\mathrm{P}$. Lee, Joo, Stark, and Chernyshenko (2019). Similarly, the averaged CORR across nonadaptive tetrad tests was .90, as opposed to .94 for tetrad CAT. In addition, CAT outperformed in both theta correlation conditions (theta correlation $=0$ and .5 ). As expected, more accurate theta estimates were found as the number of items administered increased. Taken together, our follow-up study demonstrated that the proposed MFC CAT algorithm in our study also works well with the highdimension test (i.e., ten dimensions).

\section{Discussion and conclusion}

This research has attempted to contribute to the MFC literature by exploring the effectiveness of CAT with triplet and tetrad formats using the recently developed GGUM-RANK model and information functions (Hontangas et al., 2015; Joo et al., 2018; P. Lee, Joo, Stark, \& Chernyshenko, 2019). We compared the accuracy and precision of latent trait estimation for adaptive and nonadaptive MFC pair, triplet, and tetrad tests having different percentages of unidimensional items and different correlations between latent dimensions.

The simulation results confirmed that MFC CATs outperformed nonadaptive testing on all analysis criteria (BIAS, RMSE, PSD, CORR, and REL) for MFC pair, triplet, and tetrad formats. This finding is encouraging for practitioners and researchers, because although CAT has been implemented in many testing situations, including personality assessment (e.g., Borman et al., 2001; Drasgow et al., 2012; Houston et al., 2006; Stark et al., 2014), there have been no published studies on MFC CAT with formats other than pairs. In the present study, we developed a more generalized MFC CAT using the GGUM-RANK model and further investigated the efficiency of the developed MFC CAT via a simulation study. The new development of MFC CAT could make an important contribution to noncognitive assessment by combining the benefits of CAT and MFC testing with pair, triplet, and tetrad scoring.

In addition, and more importantly, we found that less complex MFC format CATs provided results similar to or better than those from more complex nonadaptive MFC tests. From the simulation results, we found that, with the same test length and percentage of unidimensional items, pair and triplet CATs produced estimates similar to or better than nonadaptive triplet and tetrad tests, respectively. This is an important finding, because the previous studies showed that MFC triplets and tetrads generally provide better estimates than MFC pairs in nonadaptive testing (Joo et al., 2018; P. Lee, Joo, Stark, \& Chernyshenko, 2019). Our finding is encouraging for practitioners who must weigh the potential advantages and disadvantages of different MFC formats. Although using triplets and tetrads might improve accuracy and precision relative to pair measures of equal length, one must consider the potential disadvantages, such as longer testing time and greater fatigue for respondents. These disadvantages would reduce incremental validity in selection contexts. To reduce the potential disadvantages of complex MFC formats while maintaining similar or better measurement precision, simpler-format CATs (pairs and triplets) are recommended as an alternative.

In our simulation, we also found that the percentage of unidimensional items had little effect on GGUM-RANK scoring. This finding is consistent with previous MUPP CAT research (Stark et al., 2012). Moreover, although the previous research recommended including a small percentage of unidimensional items in MFC tests to increase latent trait estimation accuracy (Stark et al., 2005), the present study suggests that may be unnecessary, and it may, therefore, be possible to construct MFC tests with even greater resistance to response sets.

Before concluding, we acknowledge some limitations of our study and provide some suggestions for future research. First, the CAT algorithm for this simulation used maximum item information as the item selection method. However, as has been discussed in the CAT literature (e.g., Chang, 2015), the maximum item information approach tends to select only highly discriminating items, leaving potentially large numbers of items composed of statements with appropriate location (extremity) unused. Without exposure control, this would waste statements and potential MFC items that are timeconsuming and expensive to develop. It would, therefore, be 
beneficial to explore adaptations of alternative item selection methods, such as the a-stratified method (Chang \& Ying, 1999) or a-stratified with b-blocking method (Chang, Qian, \& Ying, 2001), in future GGUM-RANK CAT research. Given that the GGUM-RANK model was developed on the basis of multidimensional structure, an exposure control strategy for multidimensional IRT models proposed by Y. H. Lee, Ip, and Fuh (2008) could be adapted, as well. Second, we developed the CAT algorithm on the basis of the RANK-based MFC format. Although the MFC RANK format has shown the most accurate latent trait estimation, it might be more cognitively demanding than other MFC formats (e.g., PICK or MOLE), particularly when there are more than three response alternatives (e.g., Hontangas et al., 2015). Given that high cognitive demand could also affect reliability and validity, it would be worthwhile to develop MFC CAT algorithms for the PICK and MOLE variations and to study examinee reactions and correlations with cognitive ability measures. Finally, it would also be useful to explore whether MFC CATs with these three formats vary in their resistance to response biases in field studies. Research with human participants should investigate to what extent MFC CAT can reduce response and rater biases and test completion times while maintaining reliability, convergent, and criterion-related validity.

In closing, we believe this presentation offers insights that will help advance MFC CAT applications and improve the precision and efficiency of future noncognitive tests. It addresses three areas that were highlighted in the psychometric research recommendations in a 2015 National Research Council report on "Measuring Human Capabilities"-namely, multidimensional IRT, CAT, and MFC testing. We hope that the methodology examined in this article provides a foundation for research with other MFC measurement models, and eventually for studies with human research participants that examine how different item formats, test designs, instruction sets, and aberrance detection methods affect reliability and validity for institutional and organizational decision making.

\section{References}

Andrich, D. (1995). Hyperbolic cosine latent trait models for unfolding direct responses and pair- wise preferences. Applied Psychological Measurement, 19, 269-290.

Anguiano-Carrasco, C., MacCann, C., Geiger, M., Seybert, J. M., \& Roberts, R. D. (2015). Development of a forced-choice measure of typical-performance emotional intelligence. Journal of Psychoeducational Assessment, 33, 83-97.

Baker, F. B., \& Kim, S. H. (2004). Item response theory: Parameter estimation techniques. New York, NY: Dekker.

Bock, R. D., \& Mislevy, R. J. (1982). Adaptive EAP estimation of ability in a microcomputer environment. Applied Psychological Measurement, 6, 431-444.
Borman, W. C., Buck, D. E., Hanson, M., Motowidlo, S. J., Stark, S., \& Drasgow, F. (2001). An examination of the comparative reliability, validity, and accuracy of performance ratings made using Computerized Adaptive Rating Scales. Journal of Applied Psychology, 86, 965-973.

Brown, A., \& Croudace, T. J. (2015). Scoring and estimating score precision using multidimensional IRT models. In S. P. Reise \& D. A. Revicki (Eds.), Handbook of item response theory modeling: Applications to typical performance assessment (pp. 307-333). New York, NY: Routledge/Taylor \& Francis Group.

Brown, A., \& Maydeu-Olivares, A. (2011). Item response modeling of forced-choice questionnaires. Educational and Psychological Measurement, 71, 460-502.

CEB. (2010). Global personality inventory -Adaptive technical manual. Thames Ditton, UK: CEB.

Chang, H. H. (2015). Psychometrics behind computerized adaptive testing. Psychometrika, 80, 1-20.

Chang, H. H., Qian, J., \& Ying, Z. (2001). a-Stratified multistage computerized adaptive testing with $\mathrm{b}$ blocking. Applied Psychological Measurement, 25, 333-341.

Chang, H. H., \& Ying, Z. (1999). A-stratified multistage computerized adaptive testing. Applied Psychological Measurement, 23, 211-222.

Chernyshenko, O. S., Stark, S., Chan, K. Y., Drasgow, F., \& Williams, B. A. (2001). Fitting item response theory models to two personality inventories: Issues and insights. Multivariate Behavioral Research, $36,523-562$.

de la Torre, J., Ponsoda, V., Leenen, I., \& Hontangas, P. (2012, April). Examining the viability of recent models for forced-choice data. Paper presented at the Meeting of the American Educational Research Association, Vancouver, British Columbia, Canada.

de la Torre, J., Stark, S., \& Chernyshenko, O. S. (2006). Markov chain Monte Carlo estimation of item parameters for the generalized graded unfolding model. Applied Psychological Measurement, 30, 216 232.

Doornik, J. A. (2009). An object-oriented matrix programming language: Ox 6. London: Timberlake Consultants Press.

Drasgow, F., Stark, S., Chernyshenko, O. S., Nye, C. D., Hulin, C. L., \& White, L. A. (2012). Development of the tailored adaptive personality assessment system (TAPAS) to support army selection and classification decisions (Tech. Rep. No. 1311). Arlington, VA: U.S. Army Research Institute for the Behavioral and Social Sciences.

Guenole, N., Brown, A. A., \& Cooper, A. J. (2018). Forced-choice assessment of work-related maladaptive personality traits: Preliminary evidence from an application of Thurstonian item response modeling. Assessment, 25, 513-526.

Aon Hewitt. (2015). 2015 Trends in global employee engagement report. Aon Corp., Lincolnshire, IL.

Hontangas, P. M., de la Torre, J., Ponsoda, V., Leenen, I., Morillo, D., \& Abad, F. J. (2015). Comparing traditional and IRT scoring of forcedchoice tests. Applied Psychological Measurement, 39, 598-612.

Hoogland, J. J., \& Boomsma, A. (1998). Robustness studies in covariance structure modeling. Sociological Methods \& Research, 26, 329-367.

Houston, J. S., Borman, W. C., Farmer, W. L., \& Bearden, R. M. (2006). Development of the Navy Computer Adaptive Personality Scales (NCAPS) (No. NPRST-TR-06-2). Millington, TN: Navy Personnel Research, Studies, and Technology Division, Bureau of Naval Personnel (NPRST/PERS-1).

Joo, S. H., Chun, S., Stark, S., \& Chernyshenko, O. S. (2019). Item parameter estimation with the general hyperbolic cosine ideal point IRT model. Applied Psychological Measurement, 43, 18-33.

Joo, S. H., Lee, P., \& Stark, S. (2017). Evaluating anchor-item designs for concurrent calibration with the GGUM. Applied Psychological Measurement, 41, 83-96.

Joo, S. H., Lee, P., \& Stark, S. (2018). Development of Information Functions and Indices for the GGUM-RANK Multidimensional 
Forced Choice IRT Model. Journal of Educational Measurement, $55,357-372$.

Kantrowitz, T. M., Dawson, C. R., \& Fetzer, M. S. (2011). Computer adaptive testing (CAT): A faster, smarter, and more secure approach to pre-employment testing. Journal of Business and Psychology, 26, 227-232.

Kantrowitz, T. M., \& Tuzinski, K. A. (2010). The ideal point model in action: How the use of computer adaptive personality scales benefits organizations. Industrial and Organizational Psychology, 3, 507510.

Lee, P., Joo, S. H., \& Lee, S. (2019). Examining stability of personality profile solutions between Likert-type and multidimensional forced choice measure. Personality and Individual Differences, 142, 1320.

Lee, P., Joo, S. H., Stark, S., \& Chernyshenko, O. S. (2019). GGUMRANK statement and person parameter estimation with multidimensional forced choice triplets. Applied Psychological Measurement, $43,226-240$

Lee, Y. H., Ip, E. H., \& Fuh, C. D. (2008). A strategy for controlling item exposure in multidimensional computerized adaptive testing. Educational and Psychological Measurement, 68, 215-232.

Luce, R. D. (2005). Individual choice behavior. Mineola, NY: Dover. (Original work published 1959)

McCloy, R. A., Heggestad, E. D., \& Reeve, C. L. (2005). A silk purse from the sow's ear: Retrieving normative information from multidimensional forced-choice items. Organizational Research Methods, $8,222-248$

Morillo, D., Leenen, I., Abad, F. J., Hontangas, P., de la Torre, J., \& Ponsoda, V. (2016). A Dominance variant under the multiunidimensional pairwise-preference framework: Model formulation and Markov chain Monte Carlo estimation. Applied Psychological Measurement, 40, 500-516.

Mulder, J., \& Van der Linden, W. J. (2009). Multidimensional adaptive testing with optimal design criteria for item selection. Psychometrika, 74, 273-296.

Pavlov, G., Maydeu-Olivares, A., \& Fairchild, A. J. (2019). Effects of applicant faking on forced-choice and Likert scores. Organizational Research Methods, 22, 710-739. https://doi.org/10.1177/ 1094428117753683

Roberts, J. S., Donoghue, J. R., \& Laughlin, J. E. (2000). A general item response theory model for unfolding unidimensional polytomous responses. Applied Psychological Measurement, 24, 3-32.

Segall, D. O. (1996). Multidimensional adaptive testing. Psychometrika, $61,331-354$

Seybert, J. (2013). A new item response theory model for estimating person ability and item parameters for multidimensional rank order responses (Doctoral dissertation). Retrieved from http:// scholarcommons.usf.edu/etd/4942.
Stark, S., Chernyshenko, O. S., \& Drasgow, F. (2005). An IRT approach to constructing and scoring pairwise preference items involving stimuli on different dimensions: The multi-unidimensional pairwise-preference model. Applied Psychological Measurement, 29, 184-203.

Stark, S., Chernyshenko, O. S., Drasgow, F., \& White, L. A. (2012). Adaptive testing with multidimensional pairwise preference items: Improving the efficiency of personality and other noncognitive assessments. Organizational Research Methods, 15, 463-487.

Stark, S., Chernyshenko, O. S., Drasgow, F., White, L. A., Heffner, T., Nye, C. D., \& Farmer, W. L. (2014). From ABLE to TAPAS: A new generation of personality tests to support military selection and classification decisions. Military Psychology, 26, 153-164.

Stark, S., Chernyshenko, O. S., Drasgow, F., \& Williams, B. A. (2006). Examining assumptions about item responding in personality assessment: Should ideal point methods be considered for scale development and scoring? Journal of Applied Psychology, 91, 25-39.

Stark, S., Chernyshenko, O. S., \& Guenole, N. (2011). Can subject matter experts' ratings of statement extremity be used to streamline the development of unidimensional pairwise preference scales? Organizational Research Methods, 14, 256-278.

Stark, S., Chernyshenko, O. S., Lee, W. C., Drasgow, F., White, L. A., \& Young, M. C. (2011). Optimizing prediction of attrition with the U.S. Army's Assessment of Individual Motivation (AIM). Military Psychology, 23, 180-201.

Stark, S., \& Drasgow, F. (1998, April). Application of an item response theory ideal point model to computer adaptive assessment of job performance. Paper presented at the 13th annual conference for the Society of Industrial and Organizational Psychology, Dallas, TX.

Underhill, C. M., Lords, A. O., \& Bearden, R. M. (2006, October). Fake resistance of a forced choice paired-comparisons personality measure. Paper presented at the Annual Meeting of the International Military Testing Association, Kingston, ON, Canada.

Wang, W. C., Qiu, X. L., Chen, C. W., Ro, S., \& Jin, K. Y. (2017). Item response theory models for ipsative tests with multidimensional pairwise comparison items. Applied Psychological Measurement, 41, 600-613.

White, L. A., \& Young, M. C. (1998). Development and validation of the Assessment of Individual Motivation (AIM). Paper presented at the Annual Meeting of the American Psychological Association, San Francisco, CA.

Zinnes, J. L., \& Griggs, R. A. (1974). Probabilistic, multidimensional unfolding analysis. Psychometrika, 39, 327-350.

Publisher's note Springer Nature remains neutral with regard to jurisdictional claims in published maps and institutional affiliations. 